\title{
An Asymptotic Approach for the Elastodynamic Problem of a Plate under Impact Loading
}

\author{
Penelope Michalopoulou ${ }^{1,2}$ and George A. Papadopoulos ${ }^{1}$ \\ ${ }^{1}$ Department of Mechanics, National Technical University of Athens, 15773 Zographou, Greece \\ ${ }^{2}$ Ministry of Civil Works, Transportation and Infrastructure, Section D8, 15 Panag. Tsaldari Street, 17610 Athens, Greece
}

Correspondence should be addressed to Penelope Michalopoulou,p.michalopoulou@gmail.com

Received 13 June 2009; Accepted 18 January 2010

Academic Editor: Rama Bhat

Copyright ( $) 2010$ P. Michalopoulou and G. A. Papadopoulos. This is an open access article distributed under the Creative Commons Attribution License, which permits unrestricted use, distribution, and reproduction in any medium, provided the original work is properly cited.

\begin{abstract}
An approach is presented for analyzing the transient elastodynamic problem of a plate under an impact loading. The plate is considered to be in the form of a long strip under plane strain conditions. The loading is taken as a concentrated line force applied normal to the plate surface. It is assumed that this line force is suddenly applied and maintained thereafter (i.e., it is a Heaviside step function of time). Inertia effects are taken into consideration and the problem is treated exactly within the framework of elastodynamic theory. The approach is based on multiple Laplace transforms and on certain asymptotic arguments. In particular, the one-sided Laplace transform is applied to suppress time dependence and the two-sided Laplace transform to suppress the dependence upon a spatial variable (along the extent of the infinite strip). Exact inversions are then followed by invoking the asymptotic Tauber theorem and the Cagniard-deHoop technique. Various extensions of this basic analysis are also discussed.
\end{abstract}

\section{Introduction}

This work introduces an asymptotic approach based on oneand two-sided Laplace transforms to deal with the transient problem of a plate under impact. The plate is taken in the form of a long strip under plane strain conditions. A Green's function type of loading is taken here, that is, a concentrated line force (applied normal to the plate surface) with a Heaviside step function time-dependence. In this way, the present solution can be used as a fundamental solution for more general loadings. Inertia effects are taken into consideration and the problem is treated exactly within the framework of elastodynamic theory. Our main concern is on the stress and displacement field beneath the point of application of the impact load.

The present work deals with transient wave propagation in plate and strips. Related problems were examined previously by Mencher [1], Davids [2], Miklowitz [3, 4], Rosenfeld and Miklowitz [5], Lloyd and Miklowitz [6], Shmuely [7], and Dai and Wong [8], among others. Contrary to the involved analyses existing in the literature, we aim here at a simple asymptotic approach that can readily be applied to specific problems. This approach was effected by applying the Tauber theorem $[9,10]$ for asymptotic Laplace transform inversions and the Cagniard-deHoop technique $[4,11]$. We should note that for static problems inversions using the Tauber theorem were provided by Georgiadis and Papadopoulos $[12,13]$ in dealing with problems in elastic strips. The present approach may have an advantage over more involved analyses in dealing with a nonstandard material response, such as anisotropic, viscoelastic, or thermoelastic.

Analyses of problems dealing with transient wave propagation in long strips usually aim at furnishing basic theoretical information for determining scabbing effects in plates. Since this type of damage is caused by the mode conversion of the initial compressional wave into a tensile one at the free boundary of the plate, it is necessary for the analysis to study, up to a certain time, the superposition effect of multiple wave reflections at the free boundaries of the strip. In addition, another application of the transient wave propagation problem in a strip is the so-called dynamic tear test of fracture specimens $[14,15]$. This configuration involves a cracked strip, with the crack in the form of 


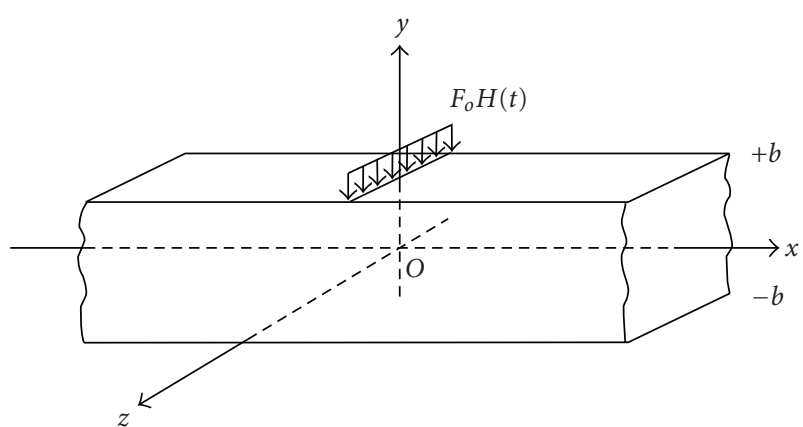

FIGURE 1: A plate or strip under the action of a concentrated impact load.

an initial notch normal to the lower side of the strip and with the impact load applied on the upper side. Experimental evidence shows that crack initiation occurs after several wave reflections (on the two sides of the strip) reach the tip of the notch [16]. Of course, as a first step of an analysis it would be helpful for one to detect the stress and displacement field in the vertical cross-section of an uncracked plate. In fact, it is this field that would be removed by the initial notch.

\section{Governing Equations and Problem Statement}

Consider a 3D elastic body in the form of a plate or strip (see Figure 1). A Cartesian coordinate system $(x, y, z)$ is used with its origin taken beneath the position of the applied loading. The body has large dimensions along the $x$ and $z$ axes as compared to the strip height $2 b$. Plane-strain conditions are assumed to prevail. The line load acts in the $(y, z)$-plane on the upper side of the plate $(y=+b)$, with an intensity $F_{0}$ (this intensity is expressed in dimensions of [force][length] ${ }^{-1}$ ) and the Heaviside step-function type dependence upon the time $t$. This means that the loading is concentrated and is applied suddenly. Since we are concerned with a solution at relatively small times, the supports of the plate do not play a role. This is a standard assumption in this type of problems $[2,3,5,7,8]$. Indeed, experimental evidence with this configuration [17] shows that the strip or plate does not bend until the elastic waves in the body reach the supports.

The transient elastodynamic response of this body is governed by the following equations (Miklowitz [4]):

$$
\begin{gathered}
\boldsymbol{\sigma}=\mu(\nabla \mathbf{u}+\mathbf{u} \nabla)+\lambda(\nabla \cdot \mathbf{u}) \mathbf{1} \\
\mu \nabla^{2} \mathbf{u}+(\lambda+\mu) \nabla(\nabla \cdot \mathbf{u})=\rho \frac{\partial^{2} \mathbf{u}}{\partial t^{2}}
\end{gathered}
$$

where (1) is the isotropic elastic constitutive law relating the stress tensor $\boldsymbol{\sigma}$ with gradients of the displacement vector $\mathbf{u}$, and (2) is the Navier-Cauchy system of partial differential equations. In the above equations, $\nabla$ is the gradient operator, $\nabla^{2}$ is the Laplace operator, $\mathbf{1}$ is the identity tensor, $\rho$ is the mass density, and $(\lambda, \mu)$ are the Lamé constants of the material. The latter constants are related to the engineering constants of the material as follows: $G \equiv \mu$ (shear modulus), $E=\mu(2 \mu+3 \lambda) /(\mu+\lambda)$ (modulus of elasticity), and

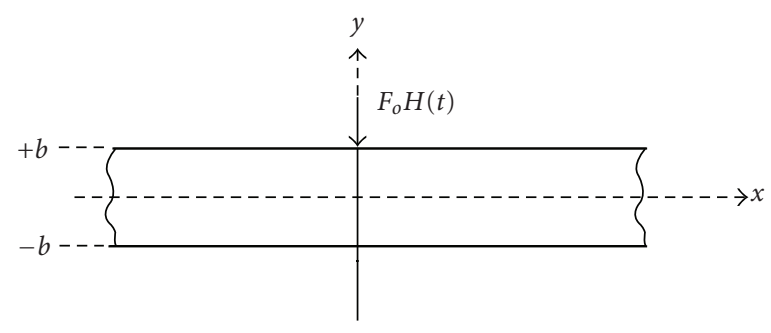

FIGURE 2: The plane-strain problem of a strip under the action of a concentrated impact load.

$\nu=\lambda / 2(\mu+\lambda)$ (Poisson's ratio). Of course, only two of the three constants $(G, E, \nu)$ are independent.

All field quantities above are functions of $(x, y, z, t)$ in a 3D problem, but here the geometry of the body and the loading conditions suggest a plane-strain problem, where the field quantities are functions of $(x, y, t)$ and are completely independent of the coordinate $z$. Therefore, we treat the 2D problem depicted in Figure 2.

Then, the displacement field takes the general form

$$
u_{x} \equiv u_{x}(x, y, t), \quad u_{y} \equiv u_{y}(x, y, t), \quad u_{z} \equiv 0,
$$

where the displacement components $\left(u_{x}, u_{y}\right)$ do not vanish identically. The in-plane stresses $\left(\sigma_{x x}, \sigma_{x y}, \sigma_{y y}\right)$ are written in terms of the displacement components as (cf. (1))

$$
\begin{gathered}
\sigma_{x x}=(\lambda+2 \mu) \frac{\partial u_{x}}{\partial x}+\lambda \frac{\partial u_{y}}{\partial y}, \\
\sigma_{x y}=\mu\left(\frac{\partial u_{x}}{\partial y}+\frac{\partial u_{y}}{\partial x}\right), \\
\sigma_{y y}=(\lambda+2 \mu) \frac{\partial u_{y}}{\partial y}+\lambda \frac{\partial u_{x}}{\partial x} .
\end{gathered}
$$

Further, we invoke the standard procedure to uncouple the Navier-Cauchy equations using the Helmholtz decomposition theorem and the displacement potentials $\phi(x, y, t)$ and $\psi(x, y, t)[4]$. The displacement and stress components are written in terms of the new functions (potentials) as follows:

$$
\begin{gathered}
u_{x}=\frac{\partial \phi}{\partial x}+\frac{\partial \psi}{\partial y}, \\
u_{y}=\frac{\partial \phi}{\partial y}-\frac{\partial \psi}{\partial x}, \\
\sigma_{x x}=\lambda \nabla^{2} \phi+2 \mu\left(\frac{\partial^{2} \phi}{\partial x^{2}}+\frac{\partial^{2} \psi}{\partial x \partial y}\right), \\
\sigma_{x y}=\mu\left(2 \frac{\partial^{2} \phi}{\partial x \partial y}-\frac{\partial^{2} \psi}{\partial x^{2}}+\frac{\partial^{2} \psi}{\partial y^{2}}\right), \\
\sigma_{y y}=\lambda \nabla^{2} \phi+2 \mu\left(\frac{\partial^{2} \phi}{\partial y^{2}}-\frac{\partial^{2} \psi}{\partial x \partial y}\right),
\end{gathered}
$$


whereas the field equations are now two wave equations for the functions $\phi$ and $\psi$ :

$$
\begin{aligned}
& \nabla^{2} \phi=\frac{1}{c_{L}^{2}} \frac{\partial^{2} \phi}{\partial t^{2}}, \\
& \nabla^{2} \psi=\frac{1}{c_{T}^{2}} \frac{\partial^{2} \psi}{\partial t^{2}} .
\end{aligned}
$$

In the above equations, $\nabla^{2}=\left(\partial^{2} / \partial x^{2}\right)+\left(\partial^{2} / \partial y^{2}\right)$ is the $2 \mathrm{D}$ Laplace operator, $c_{L}$ is the longitudinal (or dilatational) wave velocity, and $c_{T}$ is the transverse (or shear) wave velocity. We also define for future use the so-called slownesses, that is, the inverse quantities of the velocities. We denote by $a_{L} \equiv 1 / c_{L}$ the longitudinal wave slowness and by $a_{T} \equiv 1 / c_{T}$ the transverse wave slowness. Also, the wave velocities are given in terms of the density and the material constants as $c_{L}=[(\lambda+2 \mu) / \rho]^{1 / 2}$ and $c_{T}=(\mu / \rho)^{1 / 2}$ (see, e.g., Miklowitz [4]). Although (7) and (8) are independent of each other, coupling still arises through the traction conditions along the boundary of the body.

Now attention is focused to the specific problem and the boundary and initial conditions are stated as follows:

$$
\begin{aligned}
& \sigma_{y y}(x, y=+b, t)=-F_{0} \delta(x) H(t) \text { for }-\infty<x<\infty, \\
& \sigma_{x y}(x, y=+b, t)=0 \text { for }-\infty<x<\infty, \\
& \sigma_{y y}(x, y=-b, t)=0 \text { for }-\infty<x<\infty, \\
& \sigma_{x y}(x, y=-b, t)=0 \text { for }-\infty<x<\infty \\
& \phi(x, y, t)=\frac{\partial \phi(x, y, t)}{\partial t}=\psi(x, y, t)=\frac{\partial \psi(x, y, t)}{\partial t}=0 \\
& \text { at } t=0,
\end{aligned}
$$

where $H()$ is the Heaviside step function and $\delta()$ is the Dirac delta distribution $[9,10]$. For completeness of presentation, we note that the discontinuous Heaviside function is defined by $H(\tau-\omega)=1$ when $(\tau-\omega)>0$, and $H(\tau-\omega)=0$ when $(\tau-\omega)<0$, where $\tau$ is the variable and $\omega$ is a constant. In addition, the singular delta distribution has the basic property $\int_{-\infty}^{\infty} \delta(\tau-\omega) f(\tau) d \tau=f(\omega)$, where $f($ ) is a regular distribution (a function in the usual sense). We also notice that the initial conditions in (10) are pertinent to a quiescent past.

Finally, the following finiteness condition at remote regions must be satisfied $\lim _{x \rightarrow \pm \infty}(\phi, \psi, \partial \phi / \partial x, \ldots)=0$ for $-b \leq y \leq b$ and $t>0$. In fact, such a condition guarantees that the field of reflected waves consists of outgoing waves only. This condition is supported by observing the pattern of waves in Figure 3. The construction of the pattern of waves follows the basic waveguide analysis of Rosenfeld and Miklowitz [5].

The objective of the present work is to determine asymptotically the stress and displacement field in the vicinity of the cross-section beneath the load. It is indeed this area, which suffers the most severe deformation and is susceptible to fractures (scabbing phenomenon).

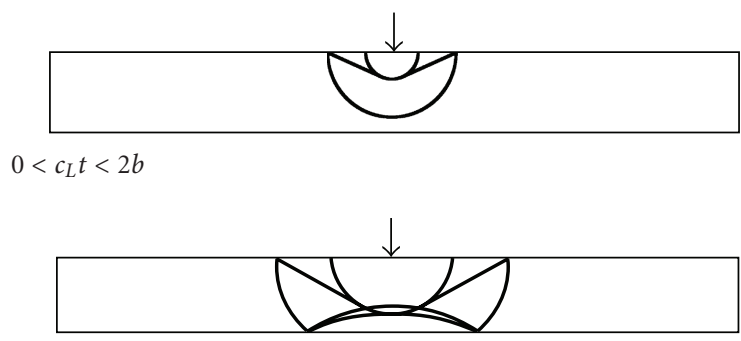

$2 b<c_{L} t<b+\left(c_{L} / c_{T}\right) b$

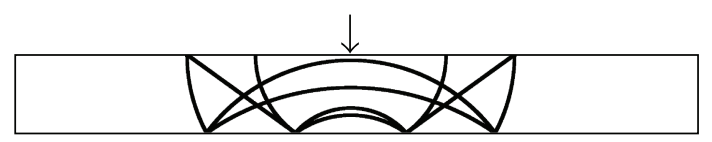

$b+\left(c_{L} / c_{T}\right) b<c_{L} t<3 b$

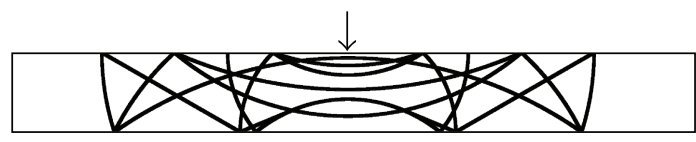

$3 b<c_{L} t<2 b+\left(c_{L} / c_{T}\right) b$

Figure 3: The pattern of reflected waves at four different time instants in the strip after the generation of the initial longitudinal and transverse waves due to the impact load.

\section{Basic Laplace-Transform Analysis}

The dependence upon $t$ and $x$ in the governing equations and the boundary/initial conditions is eliminated through the use of one- and two-sided Laplace transforms, respectively. The transforms and their corresponding inversion operations are as follows $[10,18]$ :

$$
\begin{gathered}
\bar{F}(x, y, s)=\int_{0}^{\infty} F(x, y, t) e^{-s t} d t \\
F(x, y, t)=\frac{1}{2 \pi \mathrm{i}} \int_{\mathrm{Br}} \bar{F}(x, y, s) e^{s t} d s
\end{gathered}
$$

for the one-sided transform, and

$$
\begin{aligned}
& \bar{F}^{*}(p, y, s)=\int_{-\infty}^{\infty} \bar{F}(x, y, s) e^{-s p x} d x, \\
& \bar{F}(x, y, s)=\frac{s}{2 \pi \mathrm{i}} \int_{\mathrm{Br}} \bar{F}^{*}(p, y, s) e^{s p x} d p,
\end{aligned}
$$

for the two-sided transform. In the above equations, an overbar attached in the symbol of the original function denotes its one-side Laplace transform, whereas an asterisk attached in the symbol of the one-side Laplace transform denotes its two-sided Laplace transform. In the inverse operations, $\mathrm{Br}$ denotes the Bromwich integration path (a line parallel to the imaginary axis) in the associated transform complex-planes. This path should lie within the region of analyticity of the transform. As is standard in the literature using multiple transforms for wave propagation problems $[19,20]$, the use of $s p$ (instead of $p$ alone) as the complex transform variable results in simpler algebraic 
manipulations. We also note the following [4, 9-11]. (a) Because of Lerch's theorem for the uniqueness of one-sided Laplace transforms, it is sufficient to view $\bar{F}(x, y, s)$ as a function of a real variable $s$ over some segment of the real axis in the half-plane of analyticity. Once $\bar{F}(x, y, s)$ is determined as an explicit function of $s$ in the course of solving the transformed differential equations, its definition can be extended to the whole complex s-plane, except for isolated singular points, through analytic continuation. (b) The variable $p$ should be treated as complex. (c) The twosided Laplace transform is equivalent to the more common complex Fourier transform, but its use in multiple transform analyses results in less cumbersome algebraic expressions.

Application now of the transforms (11a) and (12a) successively to (5)-(8) gives the following multiple transformed field quantities and ordinary differential equations:

$$
\begin{gathered}
\bar{u}_{x}^{*}=(s p) \bar{\phi}^{*}+\frac{d \bar{\psi}^{*}}{d y}, \\
\bar{u}_{y}^{*}=\frac{d \bar{\phi}^{*}}{d y}-(s p) \bar{\psi}^{*}, \\
\bar{\sigma}_{x x}^{*}=(\lambda+2 \mu)(s p)^{2} \bar{\phi}^{*}+\lambda \frac{d^{2} \bar{\phi}^{*}}{d y^{2}}+2 \mu(s p) \frac{d \bar{\psi}^{*}}{d y}, \\
\bar{\sigma}_{x y}^{*}=\mu\left[2(s p) \frac{d \bar{\phi}^{*}}{d y}-(s p)^{2} \bar{\psi}^{*}+\frac{d^{2} \bar{\psi}^{*}}{d y^{2}}\right], \\
\bar{\sigma}_{y y}^{*}=\lambda(s p)^{2} \bar{\phi}^{*}+(\lambda+2 \mu) \frac{d^{2} \bar{\phi}^{*}}{d y^{2}}-2 \mu(s p) \frac{d \bar{\psi}^{*}}{d y}, \\
\frac{d^{2} \bar{\phi}^{*}}{d y^{2}}-\gamma_{L}^{2} \bar{\phi}^{*}=0, \\
\frac{d^{2} \bar{\psi}^{*}}{d y^{2}}-\gamma_{T}^{2} \bar{\psi}^{*}=0,
\end{gathered}
$$

where all transformed quantities are functions of $(p, y, s)$, and

$$
\begin{aligned}
& \gamma_{L}=s\left(a_{L}^{2}-p^{2}\right)^{1 / 2}, \\
& \gamma_{T}=s\left(a_{T}^{2}-p^{2}\right)^{1 / 2} .
\end{aligned}
$$

The behavior of the functions $\gamma_{j}$ (with $j=L, T$ ) defined above, in the cut $p$-plane, is shown in Figure 4.

The ordinary differential equations (15) and (16) have the following general solutions:

$$
\begin{aligned}
& \bar{\phi}^{*}=\Phi_{1}(s, p) \cosh \left(\gamma_{L} y\right)+\Phi_{2}(s, p) \sinh \left(\gamma_{L} y\right), \\
& \bar{\psi}^{*}=\Psi_{1}(s, p) \cosh \left(\gamma_{T} y\right)+\Psi_{2}(s, p) \sinh \left(\gamma_{T} y\right),
\end{aligned}
$$

where $\Phi_{1}, \Phi_{2}, \Psi_{1}$, and $\Psi_{2}$ are arbitrary functions, which should be determined in each specific problem of elastic wave propagation. Of course, instead of the hyperbolic functions, one could use exponential functions, but the former functions suit the present analysis better as it will become clear soon (when we discuss symmetric and antisymmetric modes). The general solution along with (6) and (7) will be utilized in subsequent analysis.

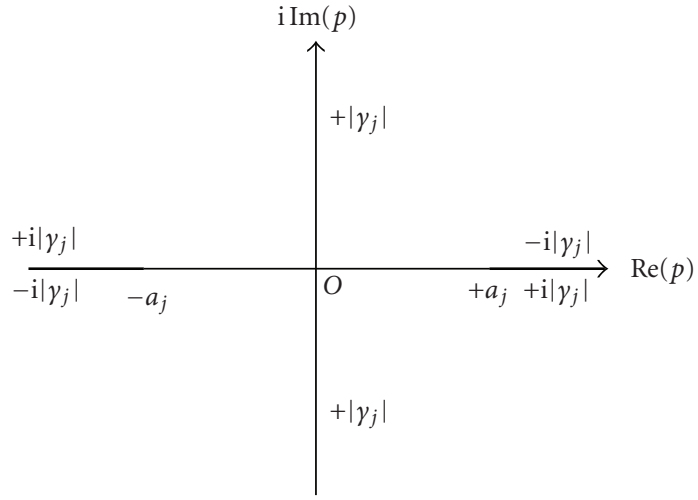

Figure 4: The complex plane with branch cuts for the functions $\gamma_{j}$.

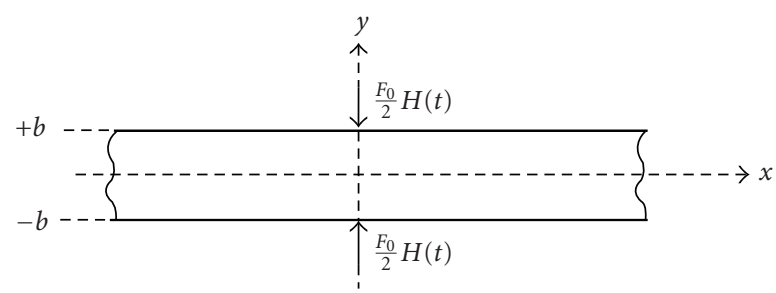

FIGURE 5: The plane-strain problem of a strip under the action of a pair of symmetric impact loads.

\section{Solution Procedure}

The solution procedure is based on superposition and asymptotic considerations.

4.1. Superposition Scheme. The solution of the problem described by (5)-(10) is greatly facilitated by decomposing the original problem into two auxiliary problems. The first (symmetric) problem and the second (antisymmetric) problem are shown in Figures 5 and 6, respectively. Clearly, the solution to the original problem results by superposing the two solutions of the auxiliary problems.

The two auxiliary problems will be analyzed separately. Both problems have the same initial conditions as the original one (cf. (10)).

The first (symmetric) problem has the following boundary conditions:

$$
\begin{gathered}
\sigma_{y y}(x, y=+b, t)=-\frac{F_{0}}{2} \delta(x) H(t) \text { for }-\infty<x<\infty, \\
\sigma_{x y}(x, y=+b, t)=0 \text { for }-\infty<x<\infty, \\
\sigma_{y y}(x, y=-b, t)=-\frac{F_{0}}{2} \delta(x) H(t) \text { for }-\infty<x<\infty, \\
\sigma_{x y}(x, y=-b, t)=0 \text { for }-\infty<x<\infty,
\end{gathered}
$$




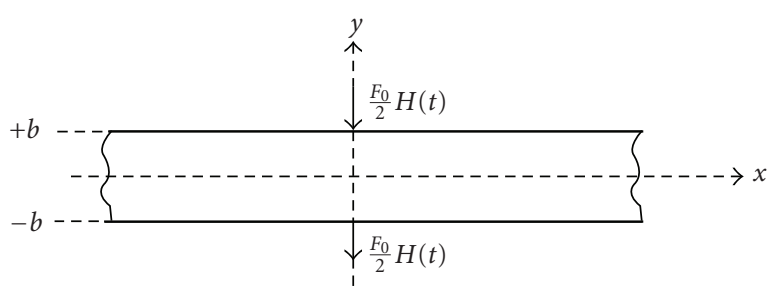

FIGURE 6: The plane-strain problem of a strip under the action of a pair of antisymmetric impact loads.

which in the multiple Laplace-transform domain become (cf. Table of transforms in [18])

$$
\begin{gathered}
\bar{\sigma}_{y y}^{*}(p, y=+b, s)=-\frac{F_{0}}{2 s}, \\
\bar{\sigma}_{x y}^{*}(p, y=+b, s)=0, \\
\bar{\sigma}_{y y}^{*}(p, y=-b, s)=-\frac{F_{0}}{2 s}, \\
\bar{\sigma}_{x y}^{*}(p, y=-b, s)=0 .
\end{gathered}
$$

In addition, due to the symmetry with respect to the line $y=0$, the following properties prevail: $u_{x}(x,-y, t)=$ $u_{x}(x, y, t)$ and $u_{y}(x,-y, t)=-u_{y}(x, y, t)$. Therefore, in view of the definition of the displacement potentials (cf. (5)), the transformed general solutions should have the following reduced forms:

$$
\begin{aligned}
& \bar{\phi}^{*}(p, y, s)=\Phi_{1}(s, p) \cosh \left(\gamma_{L} y\right), \\
& \bar{\psi}^{*}(p, y, s)=\Psi_{2}(s, p) \sinh \left(\gamma_{T} y\right) .
\end{aligned}
$$

Now, the yet unknown functions $\left(\Phi_{1}, \Psi_{2}\right)$ can be determined by inserting the expressions (21) and (22) in (14) and apply the boundary conditions in (20). Then, the following $2 \times 2$ algebraic system results

$$
\begin{aligned}
& \cosh \left(\gamma_{L} b\right)\left[\lambda(s p)^{2}+(\lambda+2 \mu) \gamma_{L}^{2}\right] \Phi_{1} \\
& -2 \mu \gamma_{T}(s p) \cosh \left(\gamma_{T} b\right) \Psi_{2}=-\frac{F_{0}}{2 s}, \\
& \sinh \left(\gamma_{T} b\right) \mu\left[\gamma_{T}^{2}-(s p)^{2}\right] \Psi_{2} \\
& +2 \mu \gamma_{L}(s p) \sinh \left(\gamma_{L} b\right) \Phi_{1}=0
\end{aligned}
$$

which when solved provides the following solution for the transformed displacement potentials:

$$
\begin{aligned}
\bar{\phi}^{*}=- & \frac{F_{0}}{2 s}\left[\sinh \left(\gamma_{T} b\right)\left(\gamma_{T}^{2}-(s p)^{2}\right) \cosh \left(\gamma_{L} y\right)\right] \\
\cdot & {\left[\sinh \left(\gamma_{T} b\right) \cosh \left(\gamma_{L} b\right)\left[\lambda(s p)^{2}+(\lambda+2 \mu) \gamma_{L}^{2}\right]\right.} \\
& \times\left(\gamma_{T}^{2}-(s p)^{2}\right)+4 \mu \gamma_{T} \gamma_{L}(s p)^{2} \\
& \left.\times \sinh \left(\gamma_{L} b\right) \cosh \left(\gamma_{T} b\right)\right]^{-1},
\end{aligned}
$$

$$
\begin{aligned}
\bar{\psi}^{*}=\frac{F_{0}}{s} & {\left[\sinh \left(\gamma_{L} b\right)\left(\gamma_{T}^{2}-(s p)^{2}\right) \sinh \left(\gamma_{T} y\right)\right] } \\
\cdot[ & \sinh \left(\gamma_{T} b\right) \cosh \left(\gamma_{L} b\right)\left[\lambda(s p)^{2}+(\lambda+2 \mu) \gamma_{L}^{2}\right] \\
& \times\left(\gamma_{T}^{2}-(s p)^{2}\right)+4 \mu \gamma_{T} \gamma_{L}(s p)^{2} \\
& \left.\times \sinh \left(\gamma_{L} b\right) \cosh \left(\gamma_{T} b\right)\right]^{-1} .
\end{aligned}
$$

Next, we record the functions $\left(\bar{\sigma}_{x x}^{*}, \bar{\sigma}_{y y}^{*}, \bar{u}_{y}^{*}\right)$ obtained by substituting (24) and (25) into (13) and (14):

$$
\begin{aligned}
\bar{\sigma}_{x x}^{*}=-\frac{F_{0}}{2 s} & {\left[\sinh \left(\gamma_{T} b\right)\left(\gamma_{T}^{2}-(s p)^{2}\right)\left[\lambda \gamma_{L}^{2}+(\lambda+2 \mu)(s p)^{2}\right]\right.} \\
& \times \cosh \left(\gamma_{L} y\right)-4 \mu \gamma_{T} \gamma_{L}(s p)^{2} \sinh \left(\gamma_{L} b\right) \\
& \left.\times \cosh \left(\gamma_{T} y\right)\right] \\
\cdot & {\left[\sinh \left(\gamma_{T} b\right) \cosh \left(\gamma_{L} b\right)\left[\lambda(s p)^{2}+(\lambda+2 \mu) \gamma_{L}^{2}\right]\right.} \\
& \times\left(\gamma_{T}^{2}-(s p)^{2}\right)+4 \mu \gamma_{T} \gamma_{L}(s p)^{2} \sinh \left(\gamma_{L} b\right) \\
& \left.\times \cosh \left(\gamma_{T} b\right)\right]^{-1},
\end{aligned}
$$

$$
\begin{aligned}
\bar{\sigma}_{y y}^{*}=-\frac{F_{0}}{2 s} & {\left[\sinh \left(\gamma_{T} b\right)\left(\gamma_{T}^{2}-(s p)^{2}\right)\left[\lambda(s p)^{2}+(\lambda+2 \mu) \gamma_{L}^{2}\right]\right.} \\
& \times \cosh \left(\gamma_{L} y\right)+4 \mu \gamma_{T} \gamma_{L}(s p)^{2} \sinh \left(\gamma_{L} b\right) \\
& \left.\quad \times \cosh \left(\gamma_{T} y\right)\right] \\
\cdot & {\left[\sinh \left(\gamma_{T} b\right) \cosh \left(\gamma_{L} b\right)\left[\lambda(s p)^{2}+(\lambda+2 \mu) \gamma_{L}^{2}\right]\right.} \\
& \times\left(\gamma_{T}{ }^{2}-(s p)^{2}\right)+4 \mu \gamma_{T} \gamma_{L}(s p)^{2} \sinh \left(\gamma_{L} b\right) \\
& \left.\times \cosh \left(\gamma_{T} b\right)\right]^{-1},
\end{aligned}
$$

$$
\begin{aligned}
\bar{u}_{y}^{*}=-\frac{F_{0}}{2 s}\left[\sinh \left(\gamma_{T} b\right) \gamma_{L}\left(\gamma_{T}^{2}-(s p)^{2}\right) \sinh \left(\gamma_{L} y\right)\right. \\
\left.+2 \gamma_{L}(s p)^{2} \sinh \left(\gamma_{L} b\right) \sinh \left(\gamma_{T} y\right)\right] \\
\cdot\left[\sinh \left(\gamma_{T} b\right) \cosh \left(\gamma_{L} b\right)\left[\lambda(s p)^{2}+(\lambda+2 \mu) \gamma_{L}^{2}\right]\right. \\
\quad \times\left(\gamma_{T}^{2}-(s p)^{2}\right)+4 \mu \gamma_{T} \gamma_{L}(s p)^{2} \sinh \left(\gamma_{L} b\right) \\
\left.\times \cosh \left(\gamma_{T} b\right)\right]^{-1} .
\end{aligned}
$$

The second (antisymmetric) problem has the following boundary conditions:

$$
\begin{gathered}
\sigma_{y y}(x, y=+b, t)=-\frac{F_{0}}{2} \delta(x) H(t) \quad \text { for }-\infty<x<\infty, \\
\sigma_{x y}(x, y=+b, t)=0 \text { for }-\infty<x<\infty, \\
\sigma_{y y}(x, y=-b, t)=\frac{F_{0}}{2} \delta(x) H(t) \quad \text { for }-\infty<x<\infty, \\
\sigma_{x y}(x, y=-b, t)=0 \text { for }-\infty<x<\infty .
\end{gathered}
$$


In this case, anti-symmetry with respect to the line $y=0$ suggests the following reduced forms for the transformed general solutions:

$$
\begin{aligned}
& \bar{\phi}^{*}(p, y, s)=\Phi_{2}(s, p) \sinh \left(\gamma_{L} y\right), \\
& \bar{\psi}^{*}(p, y, s)=\Psi_{1}(s, p) \cosh \left(\gamma_{T} y\right) .
\end{aligned}
$$

By following the same procedure as before, we get

$$
\begin{aligned}
& \bar{\phi}^{*}=- \frac{F_{0}}{2 s}\left[\cosh \left(\gamma_{T} b\right)\left(\gamma_{T}^{2}-(s p)^{2}\right) \sinh \left(\gamma_{L} y\right)\right] \\
& \cdot {\left[\sinh \left(\gamma_{L} b\right) \cosh \left(\gamma_{T} b\right)\left[\lambda(s p)^{2}+(\lambda+2 \mu) \gamma_{L}^{2}\right]\right.} \\
& \times\left(\gamma_{T}^{2}-(s p)^{2}\right)+4 \mu \gamma_{T} \gamma_{L}(s p)^{2} \sinh \left(\gamma_{T} b\right) \\
&\left.\times \cosh \left(\gamma_{L} b\right)\right]^{-1}, \\
& \bar{\psi}^{*}=\frac{F_{0}}{s}\left[\cosh \left(\gamma_{L} b\right)\left(\gamma_{T}^{2}-(s p)^{2}\right) \cosh \left(\gamma_{T} y\right)\right] \\
& \cdot\left[\sinh \left(\gamma_{L} b\right) \cosh \left(\gamma_{T} b\right)\left[\lambda(s p)^{2}+(\lambda+2 \mu) \gamma_{L}^{2}\right]\right. \\
& \quad \times\left(\gamma_{T}^{2}-(s p)^{2}\right)+4 \mu \gamma_{T} \gamma_{L}(s p)^{2} \sinh \left(\gamma_{T} b\right) \\
&\left.\quad \times \cosh \left(\gamma_{L} b\right)\right]^{-1} .
\end{aligned}
$$

For the antisymmetric problem now, the functions $\left(\bar{\sigma}_{x x}^{*}, \bar{\sigma}_{y y}^{*}, \bar{u}_{y}^{*}\right)$ are obtained by substituting (31) and (32) into (13) and (14):

$$
\begin{aligned}
\bar{\sigma}_{x x}^{*}=-\frac{F_{0}}{2 s}[ & \cosh \left(\gamma_{T} b\right)\left(\gamma_{T}^{2}-(s p)^{2}\right)\left[\lambda \gamma_{L}^{2}+(\lambda+2 \mu)(s p)^{2}\right] \\
& \times \sinh \left(\gamma_{L} y\right)-4 \mu \gamma_{T} \gamma_{L}(s p)^{2} \cosh \left(\gamma_{L} b\right) \\
& \left.\times \sinh \left(\gamma_{T} y\right)\right] \\
\cdot[ & \sinh \left(\gamma_{L} b\right) \cosh \left(\gamma_{T} b\right)\left[\lambda(s p)^{2}+(\lambda+2 \mu) \gamma_{L}^{2}\right] \\
& \times\left(\gamma_{T}^{2}-(s p)^{2}\right)+4 \mu \gamma_{T} \gamma_{L}(s p)^{2} \sinh \left(\gamma_{T} b\right) \\
& \left.\times \cosh \left(\gamma_{L} b\right)\right]^{-1}, \\
\bar{\sigma}_{y y}^{*}=-\frac{F_{0}}{2 s} & {\left[\cosh \left(\gamma_{T} b\right)\left(\gamma_{T}^{2}-(s p)^{2}\right)\left[\lambda(s p)^{2}+(\lambda+2 \mu) \gamma_{L}^{2}\right]\right.} \\
& \times \sinh \left(\gamma_{L} y\right)+4 \mu \gamma_{T} \gamma_{L}(s p)^{2} \cosh \left(\gamma_{L} b\right) \\
& \left.\quad \times \sinh \left(\gamma_{T} y\right)\right] \\
& \cdot\left[\sinh \left(\gamma_{L} b\right) \cosh \left(\gamma_{T} b\right)\left[\lambda(s p)^{2}+(\lambda+2 \mu) \gamma_{L}^{2}\right]\right. \\
& \times\left(\gamma_{T}^{2}-(s p)^{2}\right)+4 \mu \gamma_{T} \gamma_{L}(s p)^{2} \sinh \left(\gamma_{T} b\right) \\
& \left.\times \cosh \left(\gamma_{L} b\right)\right]^{-1},
\end{aligned}
$$

$$
\begin{aligned}
\bar{u}_{y}^{*}=- & \frac{F_{0}}{2 s}\left[\cosh \left(\gamma_{T} b\right) \gamma_{L}\left(\gamma_{T}^{2}-(s p)^{2}\right) \cosh \left(\gamma_{L} y\right)\right. \\
& \left.+2 \gamma_{L}(s p)^{2} \cosh \left(\gamma_{L} b\right) \cosh \left(\gamma_{T} y\right)\right] \\
\cdot & {\left[\sinh \left(\gamma_{L} b\right) \cosh \left(\gamma_{T} b\right)\left[\lambda(s p)^{2}+(\lambda+2 \mu) \gamma_{L}^{2}\right]\right.} \\
& \times\left(\gamma_{T}^{2}-(s p)^{2}\right)+4 \mu \gamma_{T} \gamma_{L}(s p)^{2} \sinh \left(\gamma_{T} b\right) \\
& \left.\times \cosh \left(\gamma_{L} b\right)\right]^{-1} .
\end{aligned}
$$

Then, the multiple transformed solution of the original problem follows by adding the solutions of the two auxiliary problems. Given the complicated forms of the above transformed expressions, it is seen that inverting the transforms is indeed a formidable task. In what follows, we concentrate on determining the field in the vicinity of the cross-section beneath the load. This is an area of intense deformation and potential fracture development.

4.2. Asymptotics and Preliminary Results. Here, we will provide an asymptotic analysis based on the Tauber theorem $[9,10]$ and present some results to check the validity of the procedure. According to the Tauber theorem, the limit of a transformed function for $|p| \rightarrow \infty$ is directly related with the limit of its original function for $|x| \rightarrow 0$. To perform such a correspondence, we will use the following asymptotic results for certain expressions entering the transformed solutions. Below, we provide details only for three typical cases and just present the remaining expressions. When $|p| \rightarrow \infty$, one obtains

$$
\begin{gathered}
\gamma_{j} \equiv s\left(\alpha_{j}^{2}-p^{2}\right)^{1 / 2}=\frac{s p}{\mathrm{i}}\left(1-\frac{\alpha_{j}^{2}}{p^{2}}\right)^{1 / 2} \\
\cong-\mathrm{i} s p\left(1-\frac{\alpha_{j}^{2}}{2 p^{2}}-\cdots\right), \\
\gamma_{L}-\gamma_{T} \cong \frac{s p}{\mathrm{i}}\left(1-\frac{a_{L}^{2}}{2 p^{2}}\right)-\frac{s p}{\mathrm{i}}\left(1-\frac{a_{T}^{2}}{2 p^{2}}\right) \\
=\frac{s}{\mathrm{i} 2 p}\left(a_{T}^{2}-a_{L}^{2}\right)=\frac{\mathrm{i} s}{2 p}\left(a_{L}^{2}-a_{T}^{2}\right), \\
4(s p)^{2} \gamma_{L} \gamma_{T}=4(s p)^{2} s^{2}\left(\alpha_{L}^{2}-p^{2}\right)^{1 / 2}\left(\alpha_{T}^{2}-p^{2}\right)^{1 / 2} \\
\cong 4(s p)^{2} s^{2}(-1) p^{2}\left(1-\frac{\alpha_{L}^{2}}{2 p^{2}}\right)\left(1-\frac{\alpha_{T}^{2}}{2 p^{2}}\right) \\
=-s^{4}\left(2 p^{2}-\alpha_{L}^{2}\right)\left(2 p^{2}-\alpha_{T}^{2}\right), \\
\gamma_{L} \gamma_{T}+(s p)^{2} \cong \frac{s^{2}}{2}\left(\alpha_{L}^{2}+\alpha_{T}^{2}\right), \\
{\left[\lambda(s p)^{2}+(\lambda+2 \mu) \gamma_{L}^{2}\right] \cong-2 \mu(s p)^{2},} \\
{\left[\lambda \gamma_{L}^{2}+(\lambda+2 \mu)(s p)^{2}\right] \cong 2 \mu(s p)^{2},}
\end{gathered}
$$

where it is noticed that writing $\left(\alpha_{j}^{2}-p^{2}\right)^{1 / 2}=(1 / \mathrm{i})\left(p^{2}-\alpha_{j}^{2}\right)^{1 / 2}$ is consistent with the specific choice of branch cuts made in Figure 4. 


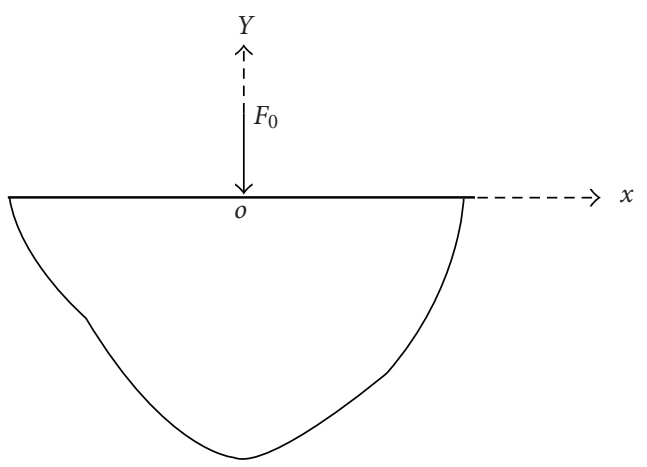

FIgURE 7: The Flamant-Boussinesq problem.

Then, the stresses $\sigma_{x x}(|x| \rightarrow 0, y=b, t)$ and $\sigma_{x x}(|x| \rightarrow$ $0, y=-b, t)$ at, respectively, the upper and lower side of the plate will be obtained. On the one hand, the former stress should tend to the expression given by the classical FlamantBoussinesq problem (the static problem of an elastic halfplane under a concentrated force at the surface; $[21,22])$. On the other hand, the latter stress should be zero because no bending of the plate exists in the infinite-strip configuration (this point was already explained in Section 2). Thus, these cases provide benchmarks for the present solution.

For the case $y=b$, the transformed solution of the original problem provides

$$
\bar{\sigma}_{x x}^{*}(|p| \longrightarrow \infty, y=b, s)=-\frac{F_{0}}{s},
$$

with the inversion (Churchill [18])

$$
\sigma_{x x}(|x| \longrightarrow 0, y=b, t)=-F_{0} \delta(x) H(t) .
$$

From the solution now of the Flamant-Boussinesq problem (see Figure 7) in a half-plane $(-\infty<x<\infty, Y<0)$ under a concentrated load, with an intensity $F_{0}$, acting at the point $(x=0, Y=0)$ of the surface (the load is directed downwards), the tangential stress $\sigma_{x x}^{\mathrm{FL}}$ (the superscript "FL" denotes that this stress follows from the Flamant-Boussinesq solution) is written as

$$
\sigma_{x x}^{\mathrm{FL}}(x, Y)=\frac{2 F_{0}}{\pi} \frac{x^{2} Y}{\left(x^{2}+Y^{2}\right)^{2}} \quad \text { for } Y<0
$$

Further, in the limit as $Y \rightarrow 0$ (which corresponds to the case $y \rightarrow b$ in the strip problem) and with the aid of the following representation of the derivative of the Dirac delta distribution (van der Pol and Bremmer [9])

$$
\delta^{\prime}(x)=\lim _{\varepsilon \rightarrow 0}\left[-\frac{2}{\pi} \frac{\varepsilon x}{\left(x^{2}+\varepsilon^{2}\right)^{2}}\right]
$$

(with $\varepsilon$ being a positive real number), we write

$$
\lim _{Y \rightarrow 0} \sigma_{x x}^{\mathrm{FL}}(x, Y)=F_{0} x \delta^{\prime}(x) .
$$

Finally, by noting that $x \delta^{\prime}(x)=-\delta(x)$ (a result from the theory of distributions_-van der Pol and Bremmer [9],
Zayed [10]), we conclude that the static Flamant-Boussinesq stress expression at the surface is indeed recovered by the present solution.

For the case $y=-b$ now, the transformed solution of the problem provides $\bar{\sigma}_{x x}^{*}(|p| \rightarrow \infty, y=-b, s)=0$. Therefore, we get $\sigma_{x x}(|x| \rightarrow 0, y=-b, t)=0$, as expected.

\section{Exact Inversion: Results}

In this section, we obtain asymptotic results by performing an exact inversion of the Cagniard-deHoop type $[4,11]$. The procedure will be presented with some details for the stress $\sigma_{x x}(|x| \rightarrow 0, y=0, t)$. Then, we also obtain the displacement $u_{y}(|x| \rightarrow 0, y=0, t)$.

By the use of (26) and (33), we first obtain the asymptotic transformed expression for the tangential stress in the original problem (note that the antisymmetric mode gives no contribution, in this case, as expected on physical grounds):

$$
\bar{\sigma}_{x x}^{*}(|p| \longrightarrow \infty, y=0, s)=-\frac{F_{0}}{2 s}\left[\frac{1}{\sinh \left(\gamma_{L} b\right)}-\frac{1}{\sinh \left(\gamma_{T} b\right)}\right] \text {. }
$$

Then, we consider the following series expansion, which is both absolutely and uniformly convergent:

$$
\begin{aligned}
\frac{1}{\sinh \left(s \kappa_{j} b\right)} & =\frac{2}{e^{s \kappa_{j} b}-e^{-s \kappa_{j} b}}=\frac{2 e^{-s \kappa_{j} b}}{1-e^{-s \kappa_{j} b}} \\
& =2 e^{-s \kappa_{j} b}\left(1+e^{-2 s \kappa_{j} b}+e^{-4 s \kappa_{j} b}+e^{-6 s \kappa_{j} b}+\cdots\right),
\end{aligned}
$$

where $\kappa_{j} \equiv \kappa_{j}(p)=\left(\alpha_{j}{ }^{2}-p^{2}\right)^{1 / 2}$ (with $j=L, T$ ), so that $\gamma_{j} \equiv s \kappa_{j}$.

Using (43) allows us to perform the inversion term by term. Still, the inversion cannot be done readily since the transforms are complicated and not available in a Table. However, the Cagniard-deHoop technique can now be applied. In short, with this technique one performs an algebraic transformation of the path in the inversion integral of the two-sided Laplace transform so that this inversion integral takes eventually the form of a recognizable onesided Laplace transform. This immediately leads to inversion by simple inspection. This technique has proven valuable in many problems of wave propagation $[4,11]$.

In view of (12b), (42), and (43), the typical inversion integral involved in the determination of the stress $\sigma_{x x}(|x| \rightarrow$ $0, y=0, t)$ is

$$
\bar{J}=\frac{1}{2 \pi \mathrm{i}} \int_{\mathrm{Br}} e^{-s\left(\kappa_{j} b-p x\right)} d p .
$$

Indeed, in the other cases corresponding to the remaining terms in (43), $s$ will be simply replaced by $3 s, 5 s, 7 s$, and so forth. To evaluate the above integral, we first perform the transformation

$$
\begin{gathered}
\left(\alpha_{j}^{2}-p^{2}\right)^{1 / 2} b-p x=t \\
\Longrightarrow p_{( \pm)}(r, \theta, t)=-\frac{t}{r} \cos \theta \pm \mathrm{i}\left(\frac{t^{2}}{r^{2}}-\alpha_{j}^{2}\right)^{1 / 2} \sin \theta,
\end{gathered}
$$




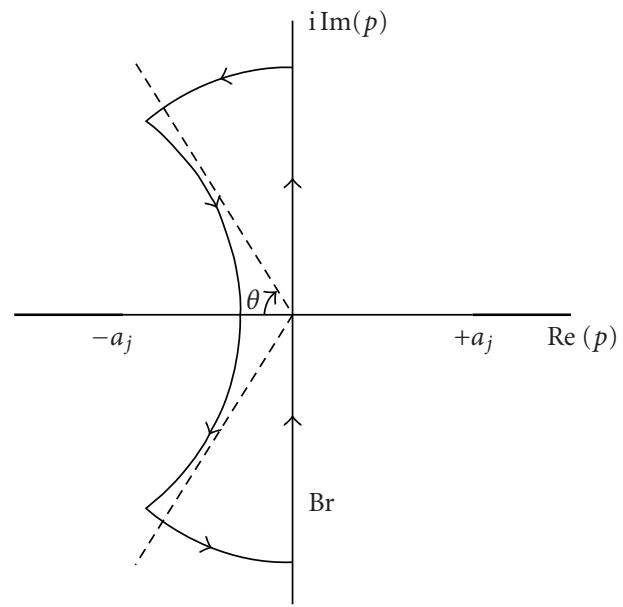

FIgURE 8: Change of contour for the inversion integral.

where $r^{2}=x^{2}+b^{2}, \tan \theta=b / x$ with $0 \leq \theta \leq \pi$. For $|x| \rightarrow 0$, we get $\cos \theta=\cos ( \pm \pi / 2)=0$.

As Figure 8 shows, (45b) expresses a hyperbola in the $p$ plane. The vertex of the hyperbola is determined to be $p=$ $-\alpha_{j} \cos \theta$ by setting the imaginary part of the RHS of (45b) equal to zero. The broken lines in Figure 8 are the asymptotes of the hyperbola. These lines are defined by

$$
\frac{\operatorname{Im}\left(p_{ \pm}\right)}{\operatorname{Re}\left(p_{ \pm}\right)}=\mp \frac{\sin \theta}{\cos \theta}=\mp \tan \theta \text {. }
$$

Notice that in this case, the transition from the original Bromwich path to the Cagniard-deHoop path (given by $(45 \mathrm{~b}))$ is simple because no poles and branch points are located inside the domain defined by these two paths. Further, if the variable $t$ is introduced along the CagniarddeHoop path, the integral in (44) will take the form

$$
\bar{J}=\frac{1}{2 \pi \mathrm{i}}\left[\int_{\infty}^{\alpha_{j} r}\left(\frac{\partial p_{(-)}}{\partial t}\right) e^{-s t} d t+\int_{\alpha_{j} r}^{\infty}\left(\frac{\partial p_{(+)}}{\partial t}\right) e^{-s t} d t\right],
$$

where

$$
\frac{\partial p_{( \pm)}}{\partial t}=-\frac{\cos \theta}{r} \pm \mathrm{i}\left(\frac{t^{2}}{r^{2}}-\alpha_{j}^{2}\right)^{-1 / 2} \frac{t}{r^{2}} \sin \theta .
$$

Then, in the limit as $|x| \rightarrow 0,(47)$ provides

$$
\lim _{|x| \rightarrow 0} \bar{J}=\frac{1}{\pi b^{2}} \int_{\alpha_{j} r}^{\infty}\left(\frac{t^{2}}{b^{2}}-\alpha_{j}^{2}\right)^{-1 / 2} t e^{-s t} d t
$$

which is now in the form of a recognizable one-sided Laplace transform; that is, it is written as $\bar{J}=\int_{\omega}^{\infty} f(t) e^{-s t} d t$. The latter form immediately leads to the inversion [18] $J=$ $f(t) H(t-\omega)$, where $\omega$ is a constant and $H()$ is the Heaviside step function. Therefore, (49) provides the inversion

$$
\lim _{|x| \rightarrow 0} J=\frac{1}{\pi b^{2}}\left(\frac{t^{2}}{b^{2}}-\alpha_{j}^{2}\right)^{-1 / 2} t H\left(t-\alpha_{j} b\right) .
$$

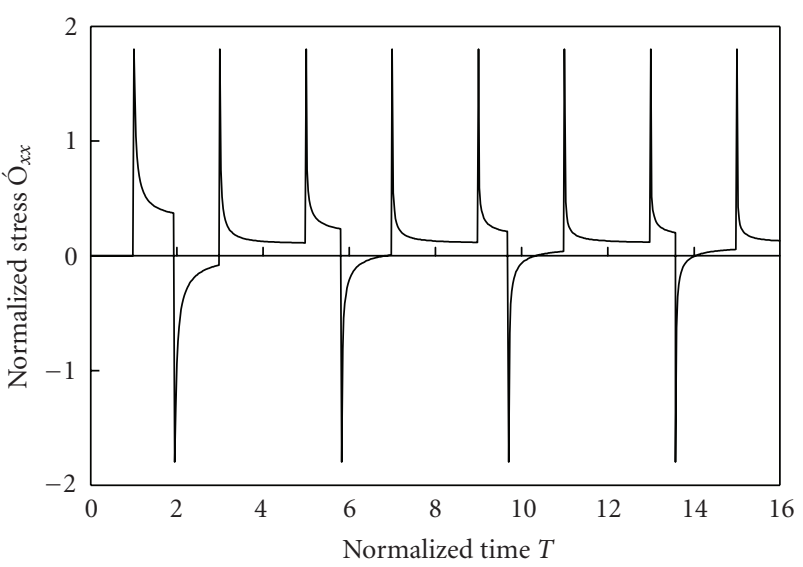

FIGURE 9: Variation of the tangential stress with time in the strip mid-plane, beneath the load.

The above technique applies also to the remaining terms in (43). Thus, results can be obtained to any desirable accuracy.

In view of the above analysis, we obtain for the stress

$$
\sigma_{x x}(|x| \longrightarrow 0, y=0, t)=-\frac{F_{0}}{\pi b^{2}}\left(Q_{L}-Q_{T}\right),
$$

where

$$
\begin{array}{r}
Q_{j}=\sum_{k=1} \frac{t}{(2 k-1)^{2}}\left[\frac{t^{2}}{(2 k-1)^{2} b^{2}}-\alpha_{j}^{2}\right]^{-1 / 2} H\left(t-a_{j}(2 k-1) b\right) \\
\text { with }(j=L, T) .
\end{array}
$$

From the above formulas, the results shown in Figure 9 are obtained by considering eight terms for $j=L$ in (52) (these terms correspond to eight arrivals of longitudinal waves in the location where the stress is calculated) and four terms for $j=T$ in (52) (these terms correspond to four arrivals of transverse waves). This choice of number of terms was made in view of the fact that the value of the velocity of longitudinal waves is roughly twice as much the value of the velocity of transverse waves, in most materials. The graph of Figure 9 depicts the variation of the normalized stress $\Sigma_{x x}=-\left(b / F_{0}\right) \sigma_{x x}$ with the normalized time $T=$ $\left(c_{L} / b\right) t$ at the location $(x=0, y=0)$. The material constants of Titanium were used here to obtain the numerical results: $\mu=43.00842 \mathrm{GPa}$ (shear modulus), $v=0.32$ (Poisson's ratio), and $\rho=4650 \mathrm{Kg} / \mathrm{m}^{3}$ (mass density). The longitudinal wave speed is $c_{L}=5900 \mathrm{~m} / \mathrm{sec}$ and the shear wave speed is $c_{T}=3041 \mathrm{~m} / \mathrm{sec}$. Finally, the strip height (plate thickness) is given a value of $2 b=0.2 \mathrm{~m}$.

One may observe in Figure 9 that the singularities correspond to the arrival of the initial longitudinal and transverse waves and their subsequent reflections at the strip boundaries. Each wave arrival at the location $(x=0, y=0)$ corresponds to the respective term in the series expansion in (52). Also, the sharp rises in Figure 9 correspond to the inverse square-root singularities in (52).

Next, we obtain the vertical displacement at the strip mid-plane $u_{y}(|x| \rightarrow 0, y=0, t)$. By the use of (28) and (35), 
we derive the following asymptotic transformed expression in the original problem:

$$
\begin{aligned}
& \bar{u}_{y}^{*}(|p| \longrightarrow \infty, y=0, s) \\
& \quad=-\frac{F_{0}}{2 \mu\left(a_{L}^{2}+a_{T}^{2}\right) s^{2}}\left[\frac{|p|}{\sinh \left(\gamma_{L} b\right)}-\frac{|p|}{\sinh \left(\gamma_{T} b\right)}\right] .
\end{aligned}
$$

It is seen from (28) that the symmetric mode gives no contribution in this case. This is to be expected on physical grounds since the displacement at the strip mid-plane $u_{y}(x, y=0, t)$ should be zero in the symmetric mode.

In view of (12b), (43), and (53), the typical inversion integral involved in the determination of the vertical displacement is

$$
\bar{I}=\frac{1}{s} \frac{1}{2 \pi \mathrm{i}} \int_{\mathrm{Br}}|p| e^{-s\left(\kappa_{j} b-p x\right)} d p .
$$

Again, the Cagniard-deHoop technique is applied. The term $(1 / s)$ before the complex integral in (54) furnishes a little complication only. Indeed, one may handle this situation by the convolution theorem for Laplace transform (see, e.g., $[10,18])$. Now, we end up with the form

$$
\bar{I}=\frac{1}{s} \int_{\omega}^{\infty} g(t) e^{-s t} d t,
$$

which when inverted (by invoking the convolution theorem) provides

$$
I=\int_{\omega}^{t} g(\tau) d \tau
$$

In the present case, we have

$$
\lim _{|x| \rightarrow 0} I=\frac{1}{2 \pi b^{2}}\left(t^{2}-\alpha_{j}^{2} b^{2}\right) H\left(t-\alpha_{j} b\right) .
$$

One can similarly obtain the integrals resulting from the remaining terms in (43). The final expression for the vertical displacement is given by

$$
u_{y}(|x| \longrightarrow 0, y=0, t)=-\frac{F_{0}}{2 \pi \mu\left(a_{L}^{2}+a_{T}^{2}\right) b^{2}}\left(W_{L}-W_{T}\right),
$$

where

$$
\begin{array}{r}
W_{j}=\sum_{k=1} \frac{\left[t^{2}-\alpha_{j}{ }^{2}(2 k-1)^{2} b^{2}\right]}{(2 k-1)^{2}} H\left(t-a_{j}(2 k-1) b\right) \\
\text { with }(j=L, T) .
\end{array}
$$

As before, representative numerical results are given for the material constants of Titanium and for a strip height $2 b=0.2 \mathrm{~m}$. The graph of Figure 10 shows the variation of the normalized vertical displacement $U_{y}=-\left(\mu / F_{0}\right) u_{y}$ with the normalized time $T=\left(c_{L} / b\right) t$ at the location $(x=0, y=0)$. It is seen that since the displacement grows indefinitely with time, the vicinity of the cross-section $(x=0)$ is a site of potential fractures due to the accumulating effect of reflected waves.

In Figure 10, the discontinuities in the slope of the displacement are due to the arrival of waves that carry singularities at their wave fronts; see Figure 9.

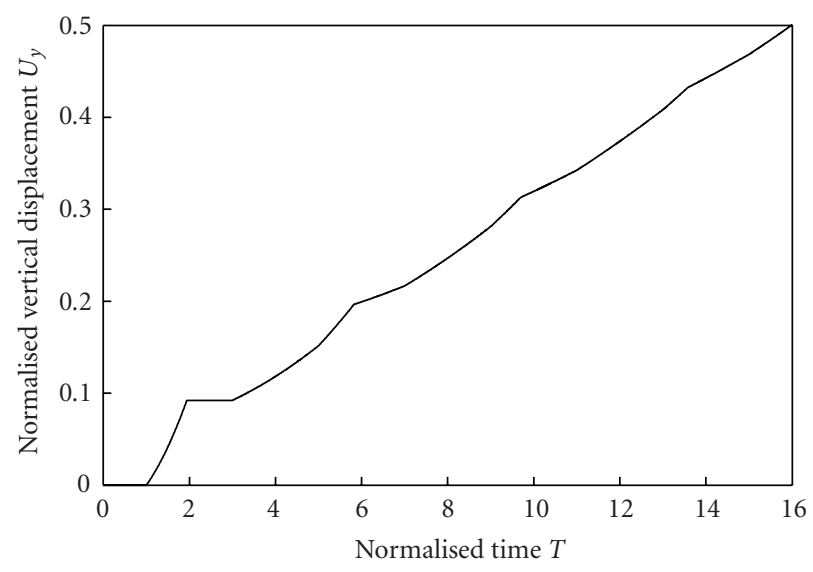

Figure 10: Variation of the vertical displacement with time in the strip mid-plane, beneath the load.

\section{Concluding Remarks}

In this paper, an approach was presented for analyzing the transient elastodynamic problem of a plate in the form of a long strip under impact. Plane strain conditions were assumed and the loading was taken as a concentrated line force applied normal to the plate surface. This force was suddenly applied and maintained thereafter. Exact elastodynamic theory was utilized in an analysis accounting for inertia effects and an explicit time dependence. Our approach was based on multiple Laplace transforms and on certain asymptotic arguments. Exact inversions were performed by invoking the asymptotic Tauber theorem and the Cagniard-deHoop technique.

Since a Green's function type of loading was taken here, the present solution can be used as a fundamental solution for more general loadings. Our analysis furnishes basic theoretical information for both determining scabbing effects in plates and studying the dynamic tear test of fracture specimens. In these applications, it is necessary for the analysis to study the superposition effect of multiple wave reflections at the free boundaries of the strip.

The asymptotic approach followed here may have an advantage over more involved analyses in dealing with a nonstandard material response such as anisotropic, viscoelastic, or thermoelastic response. Extensions of this kind are pursued by the present authors.

\section{References}

[1] A. G. Mencher, "Epicentral displacement caused by elastic waves in an infinite slab," Journal of Applied Physics, vol. 24, no. 9, pp. 1240-1246, 1953.

[2] N. Davids, "Transient analysis of stress-wave penetration in plates," Journal of Applied Mechanics, vol. 26, pp. 651-660, 1959.

[3] J. Miklowitz, "Transient compressional waves in an infinite elastic plate or elastic layer overlying a rigid half-space," Journal of Applied Mechanics, vol. 29, pp. 53-60, 1962.

[4] J. Miklowitz, The Theory of Elastic Waves and Waveguides, North-Holland, Amsterdam, The Netherlands, 1984. 
[5] R. L. Rosenfeld and J. Miklowitz, "Wave fronts in elastic rods and plates," in Proceedings of the 4th U.S. National Congress on Applied Mechanics, pp. 293-303, ASME, New York, NY, USA, 1962.

[6] J. R. Lloyd and J. Miklowitz, "On the use of double integral transforms in the study of dispersive elastic wave propagation," in Proceedings of the 4th U.S. National Congress on Applied Mechanics, pp. 255-267, ASME, New York, NY, USA, 1962.

[7] M. Shmuely, "Stress wave propagation in plates subjected to a transient line source," International Journal of Solids and Structures, vol. 11, no. 6, pp. 679-691, 1975.

[8] H.-H. Dai and R. Wong, "A uniform asymptotic expansion for the shear-wave front in a layer," Wave Motion, vol. 19, no. 3, pp. 293-308, 1994.

[9] B. van der Pol and H. Bremmer, Operational Calculus Based on the Two-Sided Laplace Integral, Cambridge University Press, Cambridge, UK, 1950.

[10] A. I. Zayed, Handbook of Function and Generalized Function Transformations, CRC Press, Boca Raton, Fla, USA, 1996.

[11] D. G. Duffy, Transform Methods for Solving Partial Differential Equations, Chapman and Hall/CRC Press, Boca Raton, Fla, USA, 2000.

[12] H. G. Georgiadis and G. A. Papadopoulos, "Determination of SIF in a cracked plane orthotropic strip by the Wiener-Hopf technique," International Journal of Fracture, vol. 34, no. 1, pp. 57-64, 1987.

[13] H. G. Georgiadis and G. A. Papadopoulos, "Cracked orthotropic strip with clampled boundaries," Journal of Applied Mathematics and Physics, vol. 39, no. 1, pp. 573-578, 1988.

[14] M. F. Kanninen and C. H. Popelar, Advanced Fracture Mechanics, Oxford University Press, New York, NY, USA, 1985.

[15] T. L. Anderson, Fracture Mechanics: Fundamentals and Applications, CRC Press, Boca Raton, Fla, USA, 1995.

[16] P. S. Theocaris, G. A. Papadopoulos, and J. Milios, "Crack interaction in bending due to impact," International Journal of Impact Engineering, vol. 2, no. 2, pp. 131-149, 1984.

[17] A. J. Durelli and W. F. Riley, "Experiments for the determination of transient stress and strain distributions in twodimensional problems," Journal of Applied Mechanics, vol. 2, no. 24, pp. 69-76, 1957.

[18] R. V. Churchill, Operational Mathematics, McGraw-Hill, Auckland, New Zealand, 1972.

[19] H. G. Georgiadis, "A correspondence principle connecting IBVPs of wave propagation and heat conduction," Journal of Applied Mathematics and Physics, vol. 43, no. 4, pp. 742-755, 1992.

[20] H. G. Georgiadis and G. Lykotrafitis, "Rayleigh waves generated by a thermal source: a three-dimensional transient thermoelasticity solution," Journal of Applied Mechanics, vol. 72, no. 1, pp. 129-138, 2005.

[21] S. P. Timoshenko and J. N. Goodier, Theory of Elasticity, McGraw-Hill, Auckland, New Zealand, 7th edition, 1982.

[22] M. H. Sadd, Elasticity, Elsevier, Amsterdam, The Netherlands, 2005. 

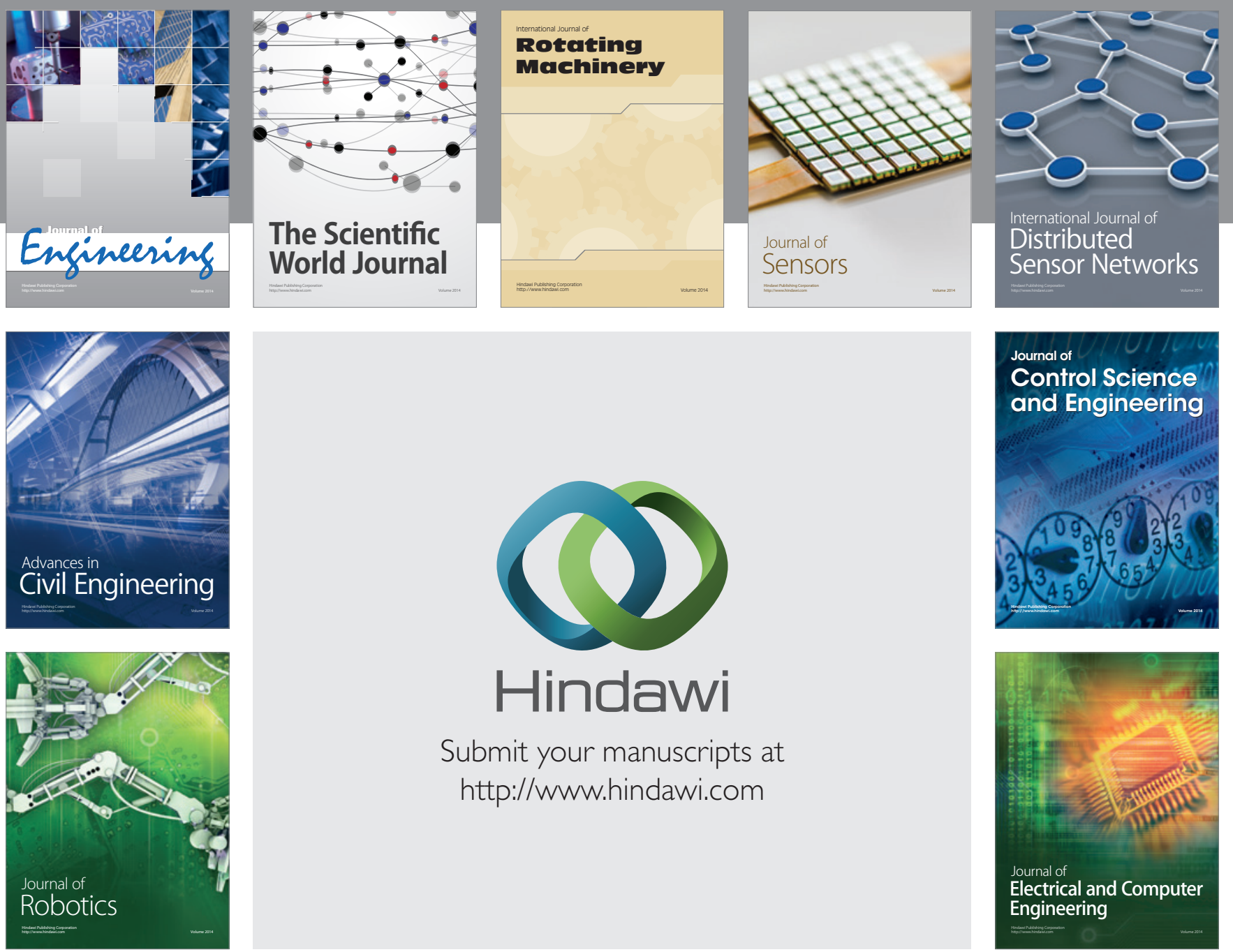

Submit your manuscripts at

http://www.hindawi.com
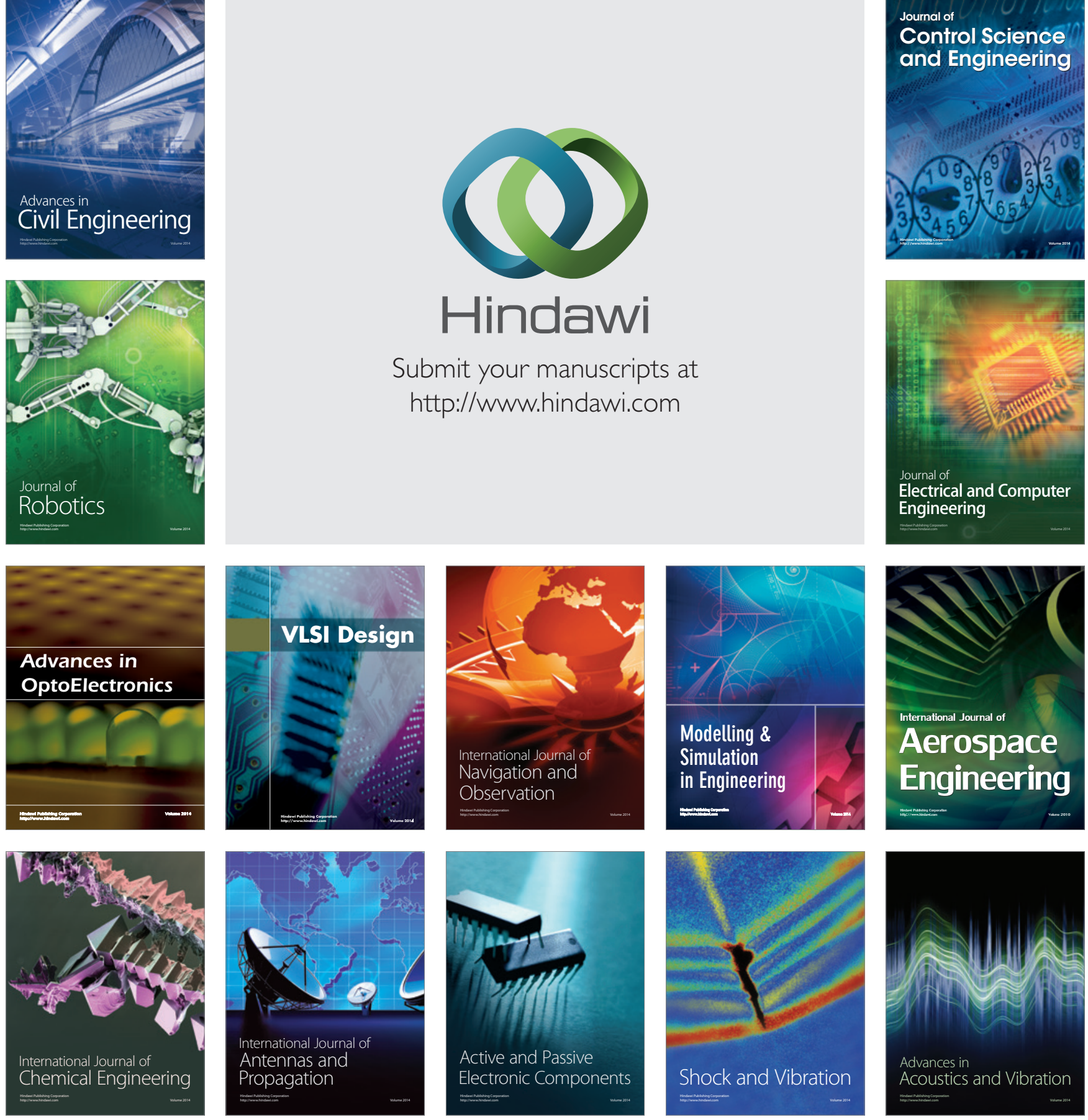